\title{
Pneumothorax and pulmonary air leaks as ventilator- induced injuries in COVID-19
}

\author{
Gabriele Martelli, Ivo Tiberio \\ Intensive Care Unit U.O.C. Anestesia e Rianimazione, Department of Surgery, University Hospital of Padua, Padua, Italy
}
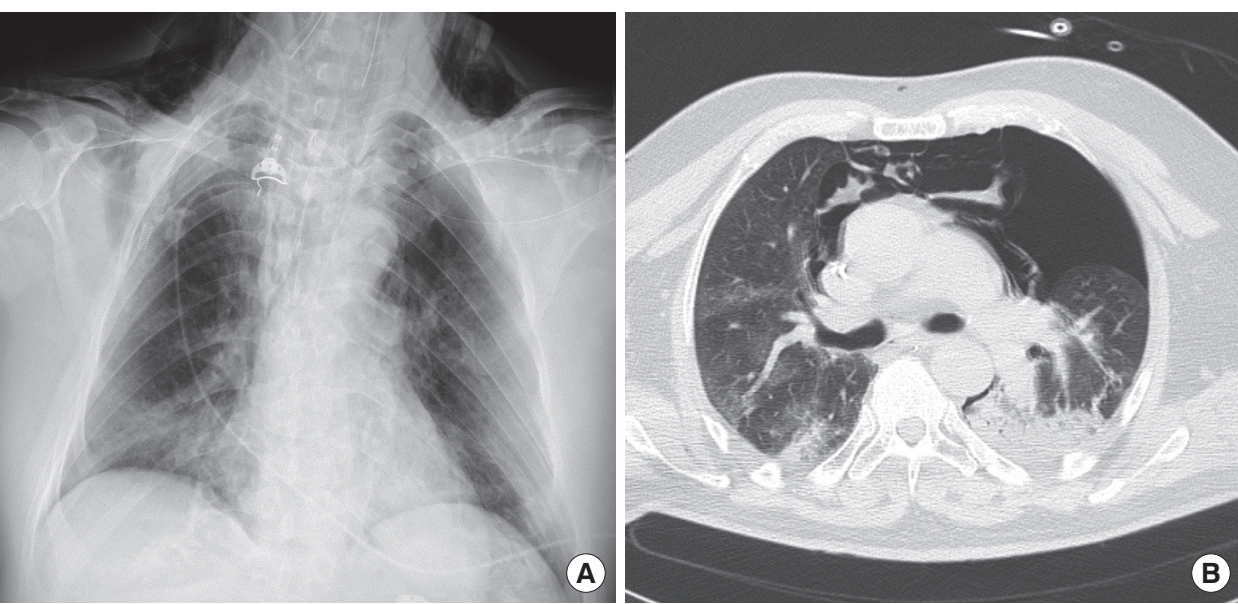

Figure 1. Chest X-ray (A) and computed tomography thoracic scan (B) of a 59-year-old male coronavirus disease 2019 (COVID-19) patient after 3 days of invasive ventilation. Ventilation occurred in pressure-control mode with the following parameters: peak inspiratory pressure, $27 \mathrm{~cm} \mathrm{H}_{2} \mathrm{O}$; positive end-expiratory pressure, $12 \mathrm{~cm} \mathrm{H} \mathrm{H}_{2} \mathrm{O}$; fraction of inspired oxygen, 0.6; inspiratory to expiratory ratio, 1:2; and respiratory rate, 16 . The last measurement prior to the occurrence of pneumothorax was a plateau pressure of $25 \mathrm{~cm} \mathrm{H}_{2} \mathrm{O}$ and static compliance of $43 \mathrm{~L} / \mathrm{cm} \mathrm{H} \mathrm{H}_{2} \mathrm{O}$. Bilateral inhomogeneous parenchyma and consolidative aspects of the left lung were noted. The patient developed left pneumothorax and pneumomediastinum. On chest X-ray, subcutaneous emphysema is evident.

Pneumothorax and other manifestations of pulmonary air leak (pneumomediastinum, subcutaneous emphysema) are well-known complications of coronavirus disease 2019 (COVID-19). The overall incidence of these complications in COVID-19 patients has been estimated to be $1 \%$ [1]. However, in mechanically ventilated COVID-19 patients, the incidence of pneumothorax and air leaks rises to $15 \%$ [2]. Despite the widespread use of protective ventilation techniques these complications remain a major concern. Severe cases of severe acute respiratory syndrome coronavirus 2 (SARS-CoV-2) pneumonia present with acute alterations such as pulmonary edema and diffuse alveolar damage [3], with a classical acute respiratory distress syndrome pattern. As a result of acute-phase alterations, there may be a negative evolution towards parenchymal consolidations and fibrosis. Due to these processes, COVID-19 patients could present with inhomogeneous pulmonary parenchyma and reduced compliance. Inhomogeneous parenchyma facilitates acute air leaks through the maldistribution of ventilatory stress (Figure 1) because two contiguous lung zones with different elastici-

\section{Image in Critical Care}

Received: November 17, 2020

Revised: December 10, 2020

Accepted: December 21, 2020

\section{Corresponding author}

Gabriele Martelli

Intensive Care Unit U.O.C. Anestesia

e Rianimazione, Department of

Surgery, University Hospital of Padua,

Via Giustiniani 235128 Padua, Italy

Tel: +39-0498212745

Fax: +39-0498218269

E-mail: gabriele.martelli@aopd. veneto.it

Copyright (@) 2021 The Korean Society of Critical Care Medicine

This is an Open Access article distributed under the terms of Creative Attributions Non-Commercial License (https:// creativecommons.org/li-censes/by-nc/4.0/) which permits unrestricted noncommercial use, distribution, and reproduction in any medium, provided the original work is properly cited. 

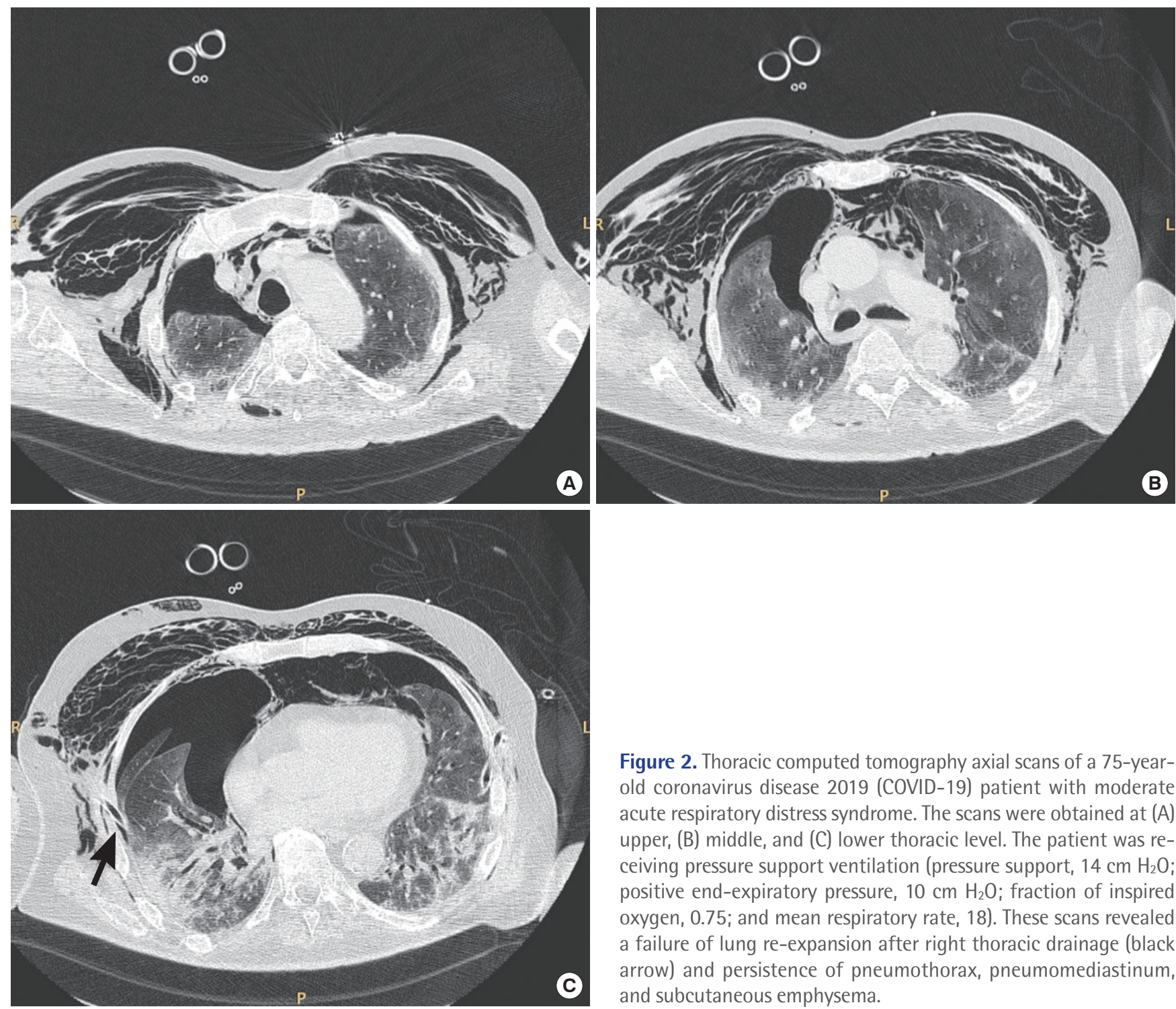

Figure 2. Thoracic computed tomography axial scans of a 75-yearold coronavirus disease 2019 (COVID-19) patient with moderate acute respiratory distress syndrome. The scans were obtained at $(A)$ upper, (B) middle, and (C) lower thoracic level. The patient was receiving pressure support ventilation (pressure support, $14 \mathrm{~cm} \mathrm{H} \mathrm{H}_{2}$; positive end-expiratory pressure, $10 \mathrm{~cm} \mathrm{H}_{2} \mathrm{O}$; fraction of inspired oxygen, 0.75 ; and mean respiratory rate, 18). These scans revealed a failure of lung re-expansion after right thoracic drainage (black arrow) and persistence of pneumothorax, pneumomediastinum, and subcutaneous emphysema.

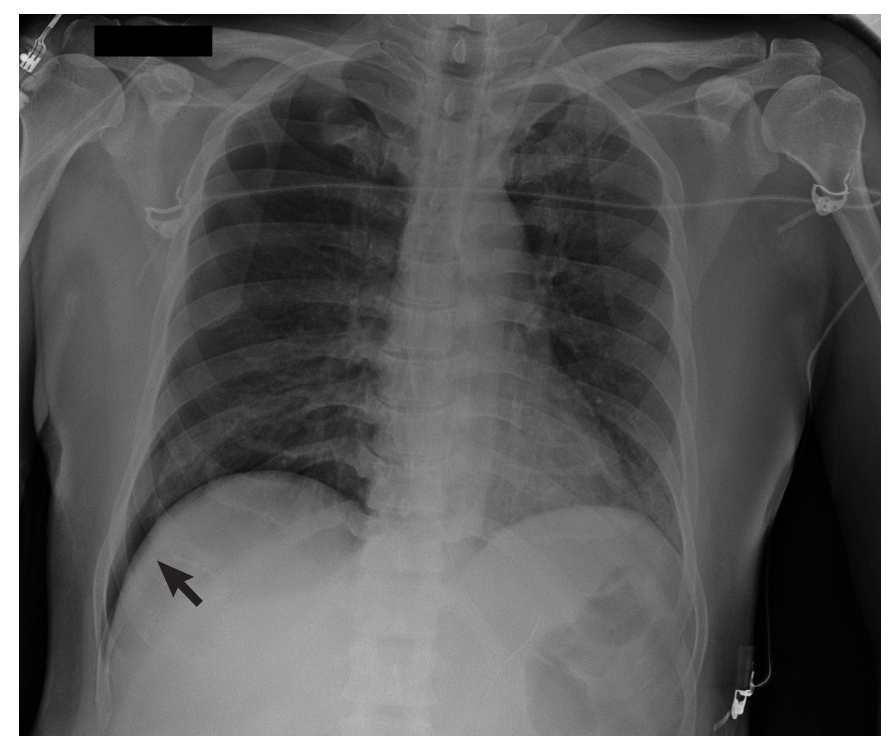

Figure 3. Chest X-ray of a 40-year-old male coronavirus disease 2019 (COVID-19) patient. Right pneumothorax of $30 \mathrm{~mm}$. "Deep sulcus sign" was noted (black arrow). This patient developed pneumothorax after a cycle of non-invasive ventilation with a helmet interface. Ventilation was set at pressure support, $8 \mathrm{~cm} \mathrm{H}_{2} \mathrm{O}$; positive end-expiratory pressure, $10 \mathrm{~cm} \mathrm{H}_{2} \mathrm{O}$; and fraction of inspired oxygen, 0.55 . 
ty develop different local stresses [4]. Reduced compliance promotes lung injury and also tends to hinder re-expansion of the lungs after air drainage (Figure 2). These factors are also involved in self-inflicted lung injury [5] and could explain the growing number of cases of pneumothorax and acute air leaks in COVID-19 patients undergoing noninvasive protective ventilation (Figure 3 ) .

\section{CONFLICT OF INTEREST}

No potential conflict of interest relevant to this article was reported.

\section{ORCID}

Gabriele Martelli https://orcid.org/0000-0001-6729-2982

\section{AUTHOR CONTRIBUTIONS}

Conceptualization, Visualization, Writing-original draft: GM. Writing-review \& editing: all author.

\section{REFERENCES}

1. Chen N, Zhou M, Dong X, Qu J, Gong F, Han Y, et al. Epidemiological and clinical characteristics of 99 cases of 2019 novel coronavirus pneumonia in Wuhan, China: a descriptive study. Lancet 2020;395:507-13.

2. Benson JC, Carlson ML, Lane JI. MRI of the internal auditory canal, labyrinth, and middle ear: how we do it. Radiology 2020;297:252-65.

3. Bradley BT, Maioli H, Johnston R, Chaudhry I, Fink SL, Xu H, et al. Histopathology and ultrastructural findings of fatal COVID-19 infections in Washington State: a case series. Lancet 2020;396:320-32.

4. Mead J, Takishima T, Leith D. Stress distribution in lungs: a model of pulmonary elasticity. J Appl Physiol 1970;28:596608.

5. Cruces P, Retamal J, Hurtado DE, Erranz B, Iturrieta P, González $\mathrm{C}$, et al. A physiological approach to understand the role of respiratory effort in the progression of lung injury in SARSCoV-2 infection. Crit Care 2020;24:494. 